\title{
Effect of Methotrexate and Tea Polyphenols on the Viability and Oxidative Stress in MDA-MB-231 Breast Cancer Cells
}

\author{
Theresa Kelly ${ }^{1}$ and Richard K. Owusu-Apenten ${ }^{1 *}$ \\ ${ }^{1}$ School of Biomedical Sciences, University of Ulster Coleraine, BT52 1SA, United Kingdom.
}

Authors' contributions

This work was carried out in collaboration between both authors. Author RKOA designed the study and wrote the protocols. Author TK managed the study, conducted the literature searches, and performed the experiments; authors TK and RKOA analysed the results. Author TK wrote the first draft of the manuscript. Both authors read and approved the final manuscript.

Article Information

DOI: $10.9734 / J A L S I / 2015 / 14142$

Editor(s):

(1) Adisorn Ratanaphan, Pharmaceutical Biotechnology, Prince of Songkla University, Thailand. (2) Sylvia Mitchell, Medicinal Plant Research Group, The Biotechnology Centre, University of the West Indies, Jamaica. Reviewers:

(1) Anonymous, México. (2) Luca Gallelli, Clinical Pharmacology, University of Catanzaro, Italy.

(3) Anonymous, India.

(4) Anonymous, Spain Complete Peer review History: http://www.sciencedomain.org/review-history.php?iid=880\&id=40\&aid=8571

\section{ABSTRACT}

Aim: To determine the effect of tea polyphenols and methotrexate on viability and reactive oxygen species (ROS) in a naturally resistant breast cancer cell line MDA-MB-231.

Methodology: MDA-MB-231 cells were selected as a model for methotrexate resistant breast cancer. Drug tests were performed over 72 hours at concentrations 0-100 $\mu \mathrm{M}$. Pre-treatments were with quercetin (QE) or epigallocatechin gallate (EGCG) for 5 hours followed by methotrexate. Cytotoxicity was measured using the MTT assay or resazurin fluorescence assay. ROS was determined using the 2', 7'-dichlorofluorescein diacetate assay. Intracellular GSH was measured using the monochlorobimane assay.

Results: Methotrexate was cytotoxic to MDA-MB-231 cells with $I C_{50}$ of $35 \pm 4 \mu \mathrm{M}$. The $I \mathrm{I}_{50}$ value was $68 \pm 9.4 \mu \mathrm{M}$ with $\mathrm{QE}$ and $83 \pm 16 \mu \mathrm{M}$ for EGCG. The pre-treatment with QE and EGCG lowered the $\mathrm{IC}_{50}$ for methotrexate by $28 \%(P=0.009)$ and $16 \% \quad(P=0.2027)$. Intracellular ROS concentrations increased after treatment with methotrexate, QE or EGCG singly and ROS 
decreased with combination treatment compared with the response for methotrexate only. There were no significant changes in intracellular GSH.

Conclusion: Pretreatment with tea polyphenols partially sensitized breast cancer cells towards methotrexate and decreases intracellular ROS. More research is needed to optimize the sensitizing effect of tea phenols on the breast cancer cell response to methotrexate.

Keywords: Breast cancer; methotrexate; quercetin; epigallocatechin gallate; oxidative stress; anticancer effect.

\section{ABBREVIATIONS}

MTT - 3-(4,5-dimethylthiazol-2-yl)-2,5-diphenyltetrazolium bromide; MTX - methotrexate; QE quercetin; EGCG - epigallocatechin gallate; ROS - reactive oxygen species; GSH - glutathione; DMEM - dulbecco's modified eagle's medium; FBS - foetal bovine serum; DMSO - dimethyl sulfoxide; MTT - thiazolyl blue tetrazolium; PBS - phosphate buffer saline; DCFH-DA - 2',7'-dichlorofluorescin diacetate; $m C B$ - monochlorobimane; $I C_{50}$ - 50\% inhibitory concentrations; RFU - relative fluorescence units; HTA - human tissue authority.

\section{INTRODUCTION}

Methotrexate was introduced in the 1950s to treat neoplastic disorders. Low dose methotrexate is used for the treatment of autoimmune disorders such as rheumatoid arthritis [1]. As a folic acid analogue, methotrexate has an antagonistic effect on folic acid metabolism that results in the inhibition of DNA / RNA synthesis and cell division [1]. The mechanism of methotrexate action is not specific for malignant cells and other cells such as the haematopoietic cells of the bone marrow, intestinal mucosa cells, and hepatocytes have been found to be particularly susceptible to methotrexate toxicity. Methotrexate treatment leads to increased myeloperoxidase, elevated reactive oxygen species (ROS) and increased oxidative stress. There is considerable interest in the use of natural agents to ameliorate methotrexate induced oxidative damage [2,3].

Breast cancer is the second most common cancer death in women within the United Kingdom; the incidence of breast cancer increased $90 \%$ between the years 1997 and 2010 [4]. The human breast cancer cell line MDA-MB-231 is oestrogen receptor negative and naturally resistant to methotrexate [5]. Methotrexate resistance in MDA-MB-231 cells is attributed to low levels ofreduced folate carrier expression [5].

Recent investigations showed that combination treatments using methotrexate and natural antioxidants decreased the levels of druginduced oxidative damage [2,3]. However, the effect of antioxidants on methotrexate effectiveness has not been widely examined. Tea polyphenols exhibit antioxidant activity and, some like epigallocatechin gallate (EGCG) showed anticancer activity [6]. Recent investigations have demonstrated that tea polyphenols enhance the susceptibility of resistant breast cancer cells to anti-estrogen therapy [7], leading to their suggested use as adjuvants in cancer therapy [8]. However, few or no studies have appeared on the effects of tea polyphenols on methotrexate-induced oxidative stress or anticancer activity. A recent investigation showed that curcumin treatment can augment methotrexate cytotoxicity [9]. The hypothesis examined in this study is that pretreatment with non-toxic doses QE or EGCG will chemosensitize breast cancer cells towards methotrexate and lower the intracellular levels of ROS. The experimental design involved assessing the dose-response for a methotrexate using a resistant breast cancer (MDA-MB-231) cell line before and after pre-treatment $Q E$ or EGCG. The effect of tea phenols pre-treatment on intracellular ROS was also monitored.

\section{MATERIALS AND METHODS}

\subsection{Cell Culture}

MDA-MB-231 cells (American Type Cell Culture) were cultured in Dulbecco's modified eagle's medium (DMEM; GIBCO) containing 10\% foetal bovine serum (FBS) (Invitrogen) in $95 \% \mathrm{O}_{2}$ and $5 \% \mathrm{CO}_{2}$ at $37^{\circ} \mathrm{C}$ (LEEC research incubator, LEEC, UK). Cells were trypsinised, counted using the NucleoCounter (NC-3000, ChemoMetec, Denmark), inoculated to give 10,000 cells/well and $50 \mu \mathrm{l}$ media in 96-microwell 
plates (Nunc; Roskilde, Denmark). The cells were then incubated overnight to allow cell attachment.

\subsection{Drug Tests}

Methotrexate $(100 \mathrm{mM}) \mathrm{QE}(100 \mathrm{mM})$ or EGCG $(40 \mathrm{mM})$ stock solutions were prepared in dimethyl sulfoxide (Sigma Aldrich) and stored at $18^{\circ} \mathrm{C}$. Each stock solution was diluted $1 / 5$ in DMEM media and filter-sterilised (Merck Millipore Ltd, Germany). Serial 1/10 dilutions of the sterile drug solutions were performed and $50 \mu$ l of the various compounds were added to inoculated wells followed by incubation for 72 hours. At the highest drug concentrations tested $(100 \mu \mathrm{M})$ the final DMSO concentration did not exceed $0.1 \%$ $(\mathrm{v} / \mathrm{v})$ which is not toxic to breast cancer cells. As required by the experimental design, first the dose-response for MDA-MB-231 was determined by treating cells with methotrexate, QE or EGCG separately at concentrations of $0-100 \mu \mathrm{M}$. From this study, the non-toxic doses of QE and EGCG were established. Non-toxic concentrations of tea phenols $(1-10 \mu \mathrm{M})$ were used to examine the effect of cell pre-treatments.

\subsection{MTT Assay for Cell Viability}

The Thiazolyl Blue Tetrazolium (MTT; SigmaAldrich) assay was performed essentially as described previously [10]. MTT (5 mg/ ml) was dissolved in phosphate buffer saline (PBS) and filter-sterilised then stored in the dark at $4^{\circ} \mathrm{C}$. Cells treated with drug were washed three times with PBS $(100 \mu \mathrm{l})$ and PBS left in the wells (100 $\mu \mathrm{l})$. MTT $(20 \mu \mathrm{L})$ was added followed by 2 hours incubation. Isopropynol-0.04N HCl solution (100 $\mu \mathrm{l})$ was added and absorbance readings were taken at $570 \mathrm{~nm}$ (VERSA Max Micro plate reader; Molecular Devices, USA).

\subsection{Resazurin Assay for Cell Viability}

Resazurin (Sigma Aldrich) solution was prepared by dissolving $1.6 \mathrm{mg}$ of resazurin in PBS (100 $\mathrm{mL}$ ). The stock solution was filter-sterilized and stored at room temperature. Pre-treatments using QE and EGCG and methotrexate drug test were performed as previously described. Cells were washed twice with ice cold PBS and resazurin was added $(10 \mu \mathrm{l})$ followed by incubation for 2 hours. Fluorescence readings were recorded with and excitation and emission wavelength ( $\lambda \mathrm{ex} / \lambda \mathrm{em}$ ) equal to $544 \mathrm{~nm} / 620-10$ nm (Fluostar Omega Fluorescence Instrument; BMG-Lab-Tech, Germany).

\subsection{Determination of Intracellular Reaction Oxygen Species}

The concentration of intracellular reactive oxygen species (ROS) was determined using 2',7'dichlorofluorescin diacetate (DCFH-DA). Breast cancer cells were seeded in microplate wells for 24 hours and pre-treated with QE or EGCG for 5 hours. The microplate wells were washed twice with media with $50 \mu \mathrm{l}$ left in the wells. Varying concentrations of methotrexate $(50 \mu \mathrm{l})$ were added to the cells followed by incubation for 72 hours. DCFH-DA $(100 \mu \mathrm{l})$ was added to the drug treated cells and cultures incubated for 2 hours. Fluorescence readings were read with $\lambda \mathrm{ex} / \lambda \mathrm{em}$ $=485 \mathrm{~nm} / 520 \mathrm{~nm}$. The reagent blank comprised cell-free microtitre plates containing $50 \mu \mathrm{l}$ media and methotrexate and the DCFH-DA assay was performed as described above. ROS were also determined using MDA-MB-231 cells pre-loaded with DCFH-DA for short-term combination treatments. Cultured cells were treated with DCFH-DA $(100 \mu \mathrm{l})$ and incubated for 1 hour. The microtitre plates were washed twice with PBS. The DCFH-preloaded cells were treated with 1 UM of methotrexate and/ or QE or EGCG for 1 hour and fluorescence readings were recording as above.

\subsection{Monochlorobimane Assay for Intracellular GSH}

Monochlorobimane (mCB) stock solution was prepared by dissolving $5 \mathrm{mg}$ of $\mathrm{mCB}$ into $2 \mathrm{~mL}$ of methanol and stored at $-18^{\circ} \mathrm{C}$. The microtitre plates were inoculated as previously described and then treated with drugs and incubated for 1 hour. The microtitre plates were then washed twice with cold PBS $(100 \mu \mathrm{l})$ and a further $100 \mu \mathrm{l}$ of PBS was left in wells. Then $5 \mu \mathrm{lmCB}$ was added to the wells and the microplate incubated for 2 hours at $37^{\circ} \mathrm{C}$. Fluorescence measurements were then recorded with $\lambda \mathrm{ex} / \lambda \mathrm{em}$ equal to 355 $\mathrm{nm} / 520 \mathrm{~nm}$.

\subsection{Statistical Analysis}

All experiments were repeated on 3 different occasions, with 6 replicates per drug concentration. Pooled data $(n=18)$ were subjected to statistical analysis. One-way ANOVA tests were performed by Microsoft SPSS version 21 to analyse statistically significant 
differences between means in conjunction with Tukey post hoc analysis for the separation of means. $P<0.05$ is considered to be statistically different. Paired t-tests performed on calculator at www.graphpad.com. To determine IC50 values, data were log-transformed and plotted versus drug concentration, followed by appropriate graphic analysis as described previously [11]. Results were identical when 1 C50 values were determined using Probit analysis.

\section{RESULTS AND DISCUSSION}

Previous research showed that QE from black tea has anti-carcinogenic and chemo-preventive properties. QE was demonstrated to reduce the viability of MDA-MB-231 cells in a dose and time dependant manner $[6,7,12,13]$; QE is indicated in the literature as a promising anti-cancer agent [13]. Epigallocatechin gallate (EGCG), which comprises $\sim 30 \%$ of the antioxidants in green tea, was found to have chemo-preventative properties [6,7]. In tests using MDA-MB-231 (ER-) cells, EGCG showed synergistic effects in combination with selective estrogen receptor modulators [7]. The aim of the current study was to examine the effect of EGCG and QE on methotrexate cytotoxicity and oxidative stress in a naturally resistant breast cancer cell line. Cell response to treatment with methotrexate, EGCG and QE alone is shown in Fig. 1.

\subsection{Effect of Methotrexate and Tea Phenols on Breast Cancer Cell Viability}

Using data from Fig. 1, the concentrations of methotrexate, QE or EGCG leading to a $50 \%$ decline in viability $\left(\mathrm{IC}_{50}\right)$ were determined for MDA-MB-231 cells treated with each agent separately, when assayed using the MTT cell proliferation assay. From Table 1 the $\mathrm{IC}_{50}$ value for methotrexate was significantly lower when compared to values for QE $(P=0.002)$ or EGCG $(P=0.006)$. There are no previous $I_{50}$ values for methotrexate, QE, or EGCG determined within a single study. However, different investigators reported the $\mathrm{IC}_{50}$ for methotrexate using MDAMB-231 cells was $80 \mu \mathrm{M}$ at 24 hours of drug treatment where a soluble analogue of MTT was the cell proliferation assay [14]. To our knowledge no previous reports using resazurin to assess methotrexate effect on MDA-MB-231 cells has been published.
Table 1. Inhibition of MDA-MB-231 breast cancer cells by methotrexate and tea phenols

\begin{tabular}{lll}
\hline MTT assay & $\mathbf{I C}_{50}\left(\times \mathbf{1 0}^{-6} \mathbf{M}\right)$ & $\boldsymbol{p}$ \\
\hline Methotrexate & $35.0 \pm 4.0^{*}$ & - \\
QE & $68.0 \pm 9.4$ & 0.002 \\
EGCG & $83.0 \pm 16$ & 0.006 \\
Resazurin assay & $\mathbf{I C}_{50}\left(\times 10^{-6} \mathrm{M}\right)$ & - \\
Methotrexate & $55.5 \pm 3.5^{*}$ & - \\
Methotrexate-QE & $40.1 \pm 4.5$ & 0.009 \\
Methotrexate- & $46.8 \pm 5.8$ & 0.203 \\
EGCG & \\
\hline \multicolumn{2}{l}{ Notes: * } \\
resazunificant difference of MTT assay or \\
= epigallocatescence results. QE = quercetin, EGCG \\
\end{tabular}

The $\mathrm{IC}_{50}$ of QE using MDA-MB-231 cells was 278 $\mu \mathrm{M}$ after 24 hours of treatment [11] whilst the $\mathrm{IC}_{50}$ value of EGCG using MDA-MB-231 cells was $87 \mu \mathrm{M}$ or $109 \mu \mathrm{M}$ for 24 or 48 hours of treatment, respectively [6]; these investigations using tea phenols and MDA-MB-231 cells employed MTT as cell proliferation assay $[6,11]$. Accordingly, past literature $\mathrm{IC}_{50}$ values based on MTT cell proliferation assay were larger compared to $\mathrm{IC}_{50}$ values in the present study probably because of the longer incubation time (72 hours) used (Table 1).

In Fig. 1, cell viability was calculated as described previously [11] using the relation; Cell viability $(\%)=\left(N_{t} / N_{0}\right)^{*} 100$, where $N_{t}$ is the cell number with drug treatment and $\mathrm{N}_{0}$ is the cell number with media only (zero drug concentration) treatment. Fig. 1 shows that low concentrations of methotrexate, QE or EGCG were stimulatory compared to $100 \%$ viability observed with cells treated with culture media only. Cytotoxicity occurred at $>10 \mu \mathrm{M}$ and methotrexate was more effective when compared to QE and EGCG (Table 1). U-shaped hormesis drug doseresponses have been reported in breast cancer cell lines treated with QE, ECGC, daidzein, resveratrol, plant phytochemicals and drugs [15]. Interestingly, we found the $\mathrm{IC}_{50}$ changed with the method for assessing residual viable cell number after drug treatment. The $I C_{50}$ value for methotrexate determined using the resazurin fluorescence assay for cell viability was $55.5 \pm 3.5$ $\mu \mathrm{M}$ and significantly different $(P<0.001)$ from the $I_{50}$ value based on the MTT assay (Table 1 ). For convenience, fluorescence instrumentation was adopted for investigations of methotrexate interactions with tea phenols (resazurin assay) as well as for monitoring changes in ROS (DCHF assay, Section 2.5). 


\subsection{Effect of Polyphenol Pre-treatment and Methotrexate on Breast Cancer Cell Viability}

Previous investigations using MDA-MB-231 demonstrated that tea phenols increase the susceptibility of MDA-MB-231 cells to certain cancer drugs [8]. In this study, MDA-MB-231 cells were pre-treated with $10 \mu \mathrm{M}$ of $\mathrm{QE}$ or ECGC for 5 hours followed by methotrexate for 72 hours. The methotrexate dose-response curves were then analysed to determine IC50 values (Table 1, lower half). In breast cancer cells pre-treated with QE there was a significant $(P<0.01)$ decline in the $I_{50}$ value for methotrexate by $28 \%$ (Table 1 ). For cells pretreated with EGCG the $\mathrm{IC}_{50}$ value for methotrexate declined by $16 \%$ but did not reach significance. These initial findings suggest tea phenols may enhance the effectiveness of methotrexate though more work will be needed to substantiate this effect.

\subsection{Methotrexate and Polyphenol Effects on Breast Cancer Cell ROS}

To get a better insight into how tea phenols affect breast cancer cells, we examined changes in intracellular levels of ROS with and without drug treatment. There were significant rises in intracellular ROS for MDA-MB-231 cells pre- treated with $10 \mu \mathrm{M}$ concentrations of $\mathrm{QE}$ or EGCG for 5 hours, followed by cell washing to remove the external phenols, and 72 hour treatment with $0-100 \mu \mathrm{M}$ of methotrexate. The results in Fig. 2 show that the concentration of ROS increased by $50 \%$ and $300 \%$ following treatment with $10 \mu \mathrm{M}$ or $100 \mu \mathrm{M}$ methotrexate compared to a non-treated control. Pre-treatment with QE or ECGC seemed to produce slightly greater rises of ROS when cells were subsequently treated with methotrexate. Under the conditions of these tests, QE or EGCG seemed to act as pro-oxidants and there was no evidence for a direct antioxidant effect.

Fig. 3 shows the changes of intracellular ROS for MDA-MB-231 cells preloaded with DCFH-DA and then treated with non-toxic doses (1 uM) of methotrexate, QE, or EGCG and combinations of these agents for 60 minutes. Interestingly, low doses of QE, EGCG and methotrexate produced increases in intracellular ROS compared with the cells exposed to culture media only (control). According to Fig. 3, the ROS levels decreased in the sequence: media $<\mathrm{MTX}+\mathrm{EGCG}=\mathrm{MTX}+\mathrm{QE}$ $<$ MTX $<$ QE < EGCG. The effect of EGCG alone was significantly higher than MTX+EGCG (Fig. 3 ). The results suggest that pre-treatment with QE or EGCG could ameliorate ROS formation in response to MTX but more research is needed to confirm this. There were no significant changes

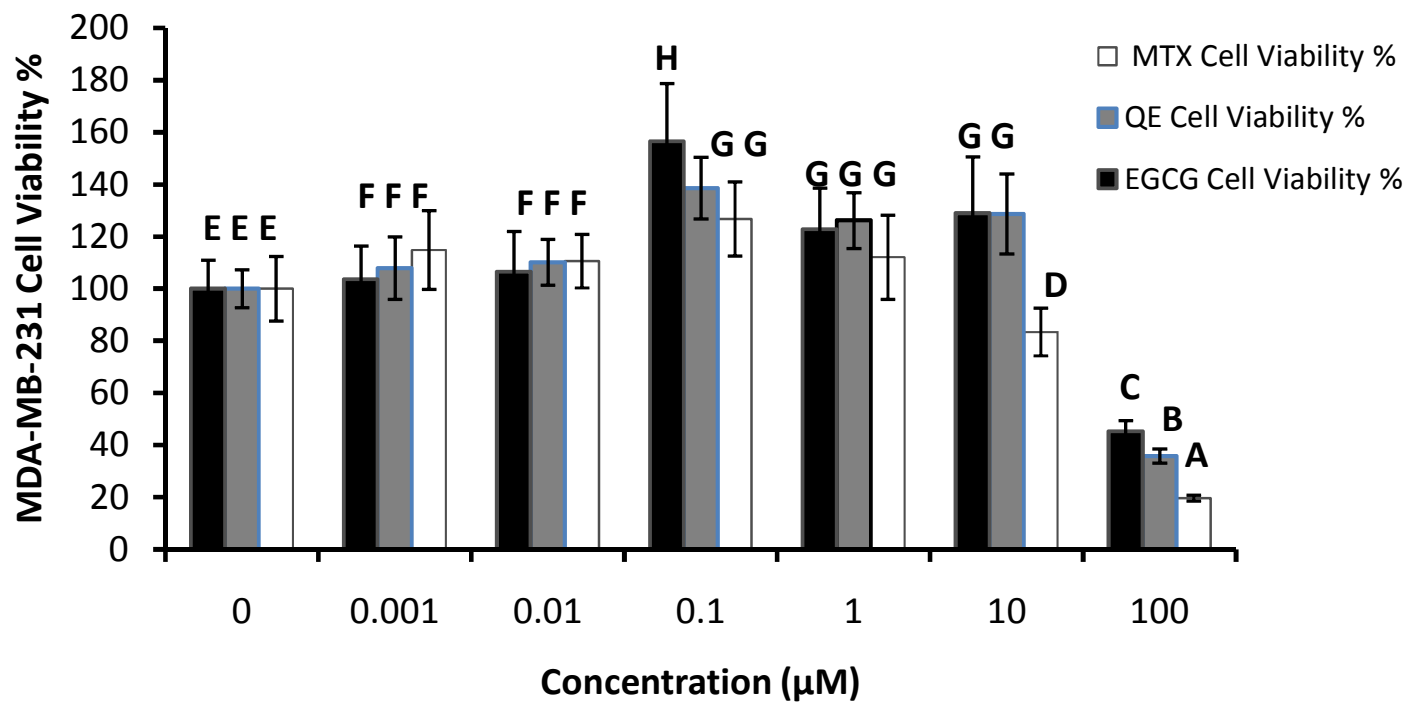

Fig. 1. The effects of methotrexate (MTX), quercetin (QE) or epigallocatechin gallate (EGCG) on MDA-MB-231 breast cancer cell viability

Bars show average values from three independent experiments with $N=18$ pooled observations with \pm standard error of the mean. Different letters at each concentration show significantly different results across all treatments in this study, $n=18$ for each treatment $(P<0.05)$. Treatment time was $72 \mathrm{hrs}$ 


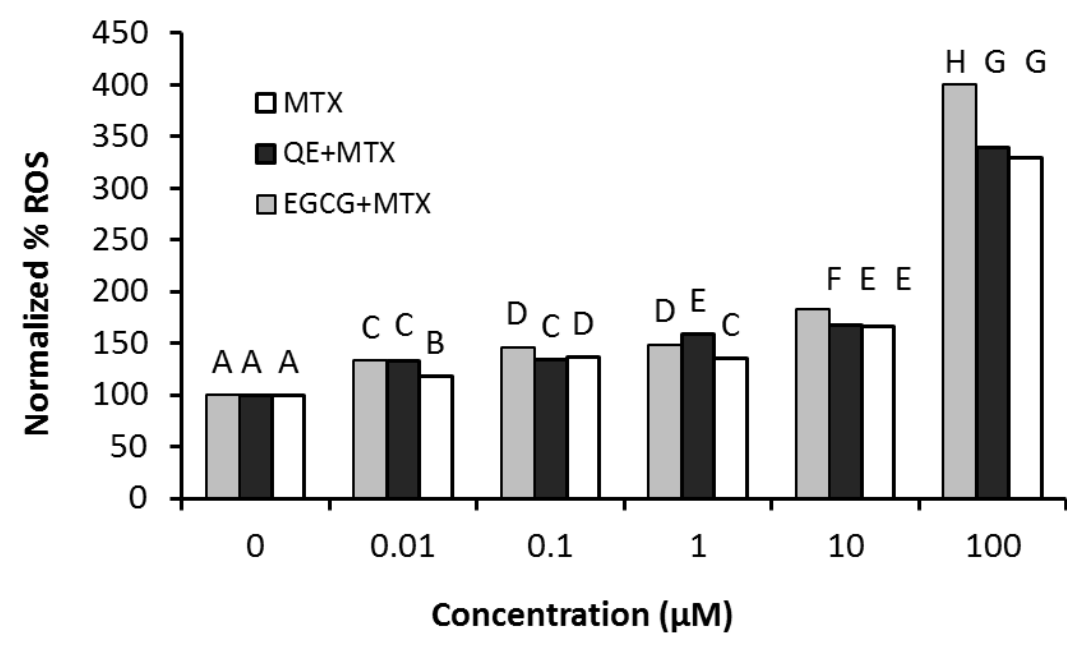

Fig. 2. The effect of breast cancer cell treatment with quercetin (QE) or epigallocatechin gallate (EGCG) on methotrexate induced reactive oxygen species (ROS) measured using 2', 7'dichlorofluorescin diacetate (DCFH-DA) as intracellular probe

Cells were treated with $10 \mu M$ of QE and EGCG for 5 hours, followed by 72 hour methotrexate drug test $n=18$ for each treatment. Different letters show significantly different results across all treatments in this study $(P<0.05)$

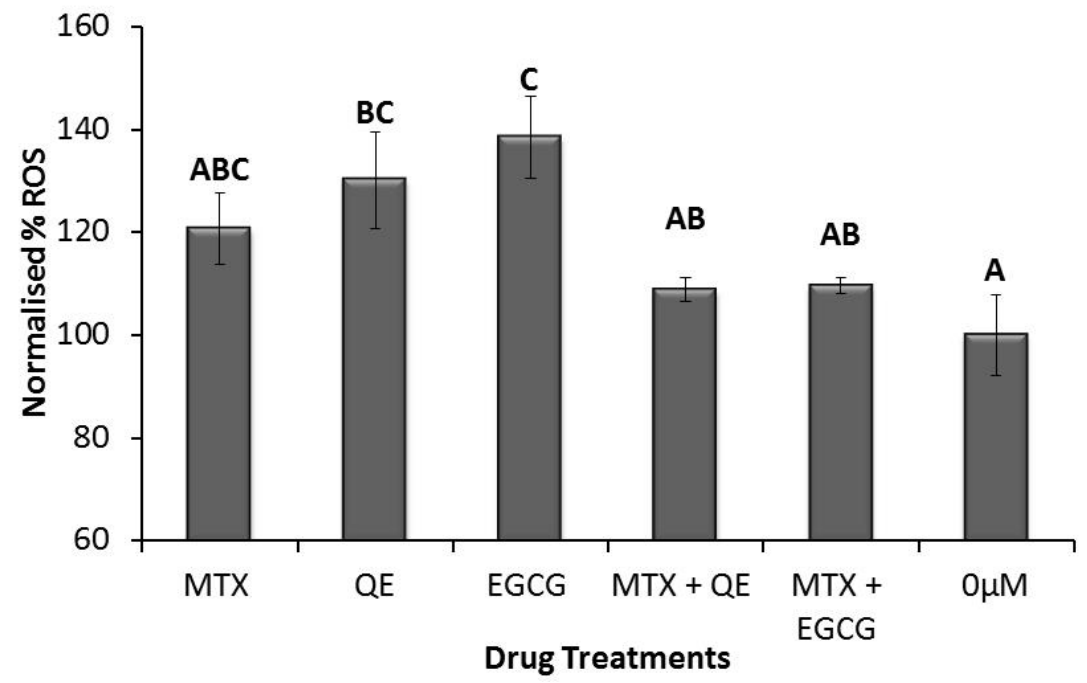

Fig. 3. The effect of combination treatment with quercetin (QE) and methotrexate or epigallocatechin gallate (EGCG) and methotrexate on intracellular reactive oxygen species (ROS) measured using 2', 7'-dichlorofluorescin diacetate (DCFH-DA) as intracellular probe MDA-MB-231 cells were treated for $60 \mathrm{~min}$. bars show average values from three independent experiments with $N=18$ pooled observations \pm standard error of the mean. Different letters at each concentration show different subsets of data identified by SPPS post-hoc analysis $(P<0.05)$

in intracellular GSH for any treatments (data not shown). Decreases of GSH may occur in cells due to rising oxidative stress. However prooxidant agents such as QE and EGCG may also increase GSH and hence the overall response is less predictable. Though plant phytochemicals behave as antioxidants in-vitro, these agents often show low levels of bioavailability and levels in the circulation or tissues are insufficient to account for a direct antioxidant effect. It has been suggested that low levels of phytochemicals and oxidation products may produce "indirect" adaptive response, .e.g. induction of antioxidant enzymes in cells [16]. 


\section{CONCLUSION}

This study confirmed that methotrexate was cytotoxic for MDA-MB-231 cells and that this cell line is more resistant compared to the nonresistant breast cancer cell lines such as MCF-7 with a reported $\mathrm{IC}_{50}$ value of $0.9 \mu \mathrm{M}$ (14). QE and EGCG are also cytotoxic to MDA-MB-231 but with lower efficacies compared to methotrexate according to their $\mathrm{IC}_{50}$ values (Table 1). A 5-hour pre-treatment with $\mathrm{QE}$ or $\mathrm{EGCG}$ prior to methotrexate decreased the $I_{50}$ value for methotrexate by $28 \%$ (QE) or $16 \%$ (EGCG) indicating tea phenols may augment the effects of methotrexate. Measurements of ROS showed methotrexate, QE and EGCG had a pro-oxidant effect in the cells. However, the rise of ROS was lower following combination treatment using methotrexate with ECGC. Under the conditions of the present study, GSH levels were not significantly affected. The initial hypothesis appears to be supported by the current finding, i.e. tea polyphenols show an enhancing effect towards methotrexate cytotoxicity that has been reported for other systems $[8,9]$.

In speculating on the wider implications of the current preclinical studies, consumption of tea is unlikely to adversely affect methotrexate therapy. However, more research is needed into the conditions required to optimize favourable interactions between tea polyphenols and methotrexate particularly since the former polyphenols are unstable under the conditions typically employed in culture [16]. In the longer term, enhancement of methotrexate by tea phenols would offer opportunities for revitalizing the use of an already established drug without further additional drug approval costs.

\section{ETHICAL APPROVAL}

MDA-MB-231 cells were cultured outside the human body; therefore the storage of cell lines does not require a human tissue authority (HTA) license. No ethical approval is required.

\section{ACKNOWLEDGEMENTS}

Mr. Stephen Kelly is thanked for supporting TK's studies in the UK. Appreciation is expressed also to the School of Biomedical Sciences, University of Ulster, for additional funding. No sponsor had a part in the study design, collection, analysis and interpretation of data or in the writing of the manuscript.

\section{COMPETING INTERESTS}

Authors have declared that no competing interests exist.

\section{REFERENCES}

1. Schmiegelow K. Advances in individual prediction of methotrexate toxicity: A review. Br J Haematol. 2009;146(5):489503.

2. Dadhania VP, Tripathi DN, Vikram A, Ramarao $P$, Jena $G B$. Intervention of alpha-lipoic acid ameliorates methotrexateinduced oxidative stress and genotoxicity: A study in rat intestine. Chem Biol Interact. 2010;183(1):85-97.

3. Cakir T, Ozkan E, Dulundu E, Topaloglu U, Sehirli AO, Ercan F, et al. Caffeic acid phenethyl ester (CAPE) prevents methotrexate-induced hepatorenal oxidative injury in rats. J Pharm Pharmacol. 2011;63(12):1566-71.

4. Breast cancer: Incidence, mortality and survival, 2010. Office for National Statistics. 2012;28:9. Accessed 31 August 2014. Available:http://www.ons.gov.uk/ons/rel/ca ncer-unit/breast-cancer-inengland/2010/sum-1.html

5. Worm J, Kirkin AF, Dzhandzhugazyan KN, Guldberg P. Methylation-dependent silencing of the reduced folate carrier gene in inherently methotrexate-resistant human breast cancer cells. J Biol Chem. 2001;276(43):39990-40000.

6. Thangapazham RL, Singh AK, Sharma A, Warren J, Gaddipati JP, Maheshwari RK. Green tea polyphenols and its constituent epigallocatechin gallate inhibit proliferation of human breast cancer cells in vitro and in vivo. Cancer Lett. 2007;245(1-2):232-41.

7. Stuart EC, Jarvis RM, Rosengren RJ. In vitro mechanism of action for the cytotoxicity elicited by the combination of epigallocatechin gallate and raloxifene in MDA-MB-231 cells. Oncol Rep. 2010;24 (3):779-85.

8. Lecumberri E, Deupertuis YM, Mralbell R, Pichard C. Green tea polyphenol epigallocatechin-3-gallate (EGCG) as adjuvant in cancer therapy. Clin Nutr. 2013; (32):894-903.

9. Dhanasekaran S. Biswal BK, Sumantran VN, Verma RS. Augmented sensitivity to methotrexate by curcumin induced overexpression of folate receptor in KG-1 cells. Biochimie. 2013;95(8):1567-73. 
10. Denizot F, Lang R. Rapid colorimetric assay for cell growth and survival. modifications to the tetrazolium dye procedure giving improved sensitivity and reliability. J Immunol Methods. 1986;89 (2):271-7.

11. Magee PJ, Owusu-Apenten R, McCann MJ, Gill Cl, Rowland IR. Chickpea (Cicer arietinum) and other plant derived protease inhibitor concentrates inhibit breast and prostate cancer cell Proliferation In vitro. Nutri Cancer. 2012;64(5):741-748.

12. Chien SY, Wu YC, Chung JG, Yang JS, Lu $\mathrm{HF}$, Tsou MF, et al. Quercetin-induced apoptosis acts through mitochondrial- and caspase-3-dependent pathways in human breast cancer MDA-MB-231 cells. Hum Exp Toxicol. 2009;28(8):493-503.
13. Murakami A, Ashida H, Terao J. Multitargeted cancer prevention by quercetin. Cancer Lett. 2008;269(2):315-25.

14. Wu Z, Shah A, Patel N, Yuan X. Development of methotrexate proline prodrug to overcome resistance by MDAMB-231 cells. Bioorg Med Chem Lett. 2010; 20(17):5108-12.

15. Calabrese EJ, Cancer biology and hormesis: Human tumor cell lines commonly display hormetic (biphasic) dose responses. Crit Rev Toxic. 2005;35(6):463582.

16. Babich $\mathrm{H}$, Schuck AG, Weisburg JH, Zuckerbraun HL. Research strategies in the study of the pro-oxidant nature of polyphenol nutraceuticals. J Toxicol. 2011;2011:467305.

(c) 2015 Kelly and Owusu-Apenten; This is an Open Access article distributed under the terms of the Creative Commons Attribution License (http://creativecommons.org/licenses/by/4.0), which permits unrestricted use, distribution, and reproduction in any medium, provided the original work is properly cited.

Peer-review history:

The peer review history for this paper can be accessed here: http://www.sciencedomain.org/review-history.php?iid=880\&id=40\&aid=8571 